\title{
A moving frame of reference algorithm for fluid/structure interaction of rotating and translating bodies
}

\author{
International Journal for Numerical Methods in Fluids \\ L. Li, ${ }^{*} \quad$ S. J. Sherwin ${ }^{*}, P$. W. Bearman \\ Department of Aeronautics, \\ Imperial College of Science, Technology and Medicine, \\ Prince Consort Road, London SW7 $2 B Y$, U.K.
}

\begin{abstract}
SUMMARY
A mathematical and numerical formulation is derived for fluid/structure interaction problems involving arbitrary geometries relevant to the simulation of bridge deck instabilities due to cross winds. A translating and rotating moving frame of reference is attached to the body to utilise an efficient fixed mesh spectral/hp element solver. The formulation is validated against experiments with flow simulations of circular cylinders at Reynolds numbers of 100-400 undergoing free and forced motion in the transverse and in-line directions. The well documented phenomena of vortex lock-in is captured. The formulation is then applied to rectangular bodies at Re $=250$ under forced and free motion the latter of which demonstrates torsional galloping. Copyright (C) 2000 John Wiley \& Sons, Ltd.
\end{abstract}

\section{Introduction}

Fluid/structure interaction problems are common in applications involving aeronautics, offshore engineering, civil and mechanical engineering. Since the beginning of the last decade a number of numerical algorithms have been developed for the solution of fluid/structure interaction problems with the aim of meeting the needs of fundamental research and engineering application. Among them are finite element methods [1, 2], based on the ALE formulation, and finite volume methods [3], also using deformable hybrid grids, and spectral element methods using coordinate transformation [4]. These algorithms were confined to the study of a body with transverse or in-line motion. However, in practice, the torsional motion of many body shapes also plays a very important role, particularly for long-span bridges. A classic example is the collapse of Tacoma Narrows bridge, which was essentially due to a torsional instability $[5,6,7]$.

\footnotetext{
*Current address: Department of Mathematics, University College London, Gower Street, London WC1E 6BT, $\mathrm{UK}$.

${ }^{*}$ Correspondence to: S.J. Sherwin, Department of Aeronautics, Imperial College, Prince Consort Road, London, SW7 2BY UK.

Contract/grant sponsor: EPSRC; contract/grant number: GR/L48096
} 
For the problem of an elastically mounted rigid body acted upon by flow forces, the governing equations are the incompressible Navier-Stokes equations with moving boundary conditions, i.e.,

$$
\begin{aligned}
\nabla \cdot \mathbf{v} & =0 \\
\frac{\partial \mathbf{v}}{\partial t}+(\mathbf{v} \cdot \nabla) \mathbf{v} & =-\nabla p+\nu \nabla^{2} \mathbf{v} \\
\mathbf{v} & =\mathbf{v}_{b}(t) \quad \text { on } \Gamma(t)
\end{aligned}
$$

Here $\mathbf{v}$ is the fluid velocity, $p$ is the reduced pressure and $\Gamma(t)$ denotes the interface between the flow and the body and moves with the unknown velocity $\mathbf{v}_{b}$. The motion of the body is governed by the structural equation symbolically written as

$$
\mathbf{L}\left(\frac{\partial^{2} \delta}{\partial t^{2}}, \frac{\partial \delta}{\partial t}, \delta\right)=f(\nu, \mathbf{v}, p)
$$

where $\mathbf{L}$ is considered as a linear operator and $\delta$ is a variable describing the motion of the body, for example, the vertical displacement and/or rotational angle of the structure, i.e., the heaving and/or torsional mode.

Instead of directly solving the coupled system (1) and (2), an efficient strategy is to solve the Navier Stokes and structural equations explicitly and thereby decouple their solutions over each time step. Therefore, at each time level, the Navier Stokes equations are solved to obtain the aerodynamic forces acting on the body. These forces are then an input to the structural solver which predicts the velocity of the body at the next time level. Finally the body velocities are then used to advance the fluid solver in a so called 'loose' coupling algorithm.

The most general technique to solve the Navier Stokes equation with moving boundary conditions is the so-called Arbitrary Lagrangian-Eulerian (ALE) method [8, 9]. In this approach the computational mesh is continually deformed, which causes the discrete Navier Stokes solver to change at each time step. Therefore it is not possible to take advantage of the optimised direct solvers for static meshes developed for many traditional applications.

An alternative approach for solving the moving-boundary flow problem for a flexibly mounted but non-deforming or rigid body is to attach the coordinate system to the body, and solve the Navier Stokes equations in a moving frame of reference. For example, in [4], a coordinate transformation

$$
x^{\prime}=x-\zeta(z, t), \quad y^{\prime}=y-\eta(z, t), \quad z^{\prime}=z, \quad t^{\prime}=t
$$

was applied to a flexible cable, where $\zeta(z, t)$ and $\eta(z, t)$ are the displacements of the cable in the $x$ and $y$-directions. The advantage of such an approach is that an optimised direct solver for the fluid can be efficiently applied. This is particularly important when considering the very long simulation time typically required to capture the instability of fluid structure interaction. However, the transformation applied in [4] excludes the important case of rotation, which is of great significance in problems such as bridge deck dynamics.

In this paper, we present our work using the moving boundary approach for a single body undergoing both translation and rotation. We formulate, from first principles, a coordinate 
transformation which attaches the coordinate system to the heaving/rotating body. This formulation proves very flexible in handling every possible motion of a body in a two dimensional plane, and it also has the potential to handle three dimensional twisting motions. The high-order splitting scheme and spectral/hp spatial discretisation adopted in the current work are then presented. Finally, numerical simulations are presented for translational, in-line and rotational modes, and it is shown that the vortex lock-in and the galloping phenomenon are successfully captured by the computations.

\section{Formulation}

Assume that instantaneously the body moves with translation $\mathbf{d}=(g(t), h(t))^{T}$ and rotational angle $\theta=\theta(t)$, in the absolute frame of reference $\left(x^{\prime}, y^{\prime}\right)$. Then a corresponding moving frame of reference can be attached on the body with the transformation

$$
\begin{aligned}
& x^{\prime}=g(t)+x \cos \theta+y \sin \theta, \\
& y^{\prime}=h(t)-x \sin \theta+y \cos \theta .
\end{aligned}
$$

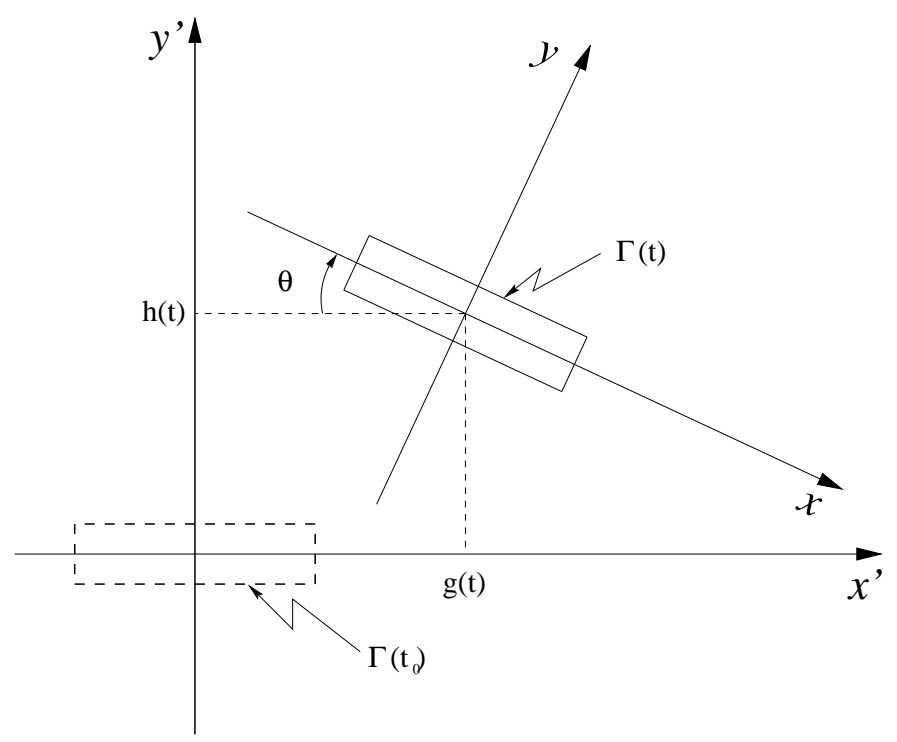

Figure 1. Coordinate transformation.

Here the prime coordinate system is used to denote the absolute frame of reference. The coordinates $\mathbf{x}=(x, y)^{T}$ denotes the moving frame of reference whilst $\mathbf{d}=(g(t), h(t))^{T}$ is the coordinate of the origin of the moving reference in the absolute frame of reference. The rotational angle $\theta(t)$ is defined to be consistent with the aeronautical sign convention for angle of attack, i.e., rotating the model clockwise in a flow from left to right increases the angle, see figure 1. 


\subsection{Transforming the Navier Stokes Equations}

We have the following basic relations in matrix/vector notation

$$
\mathbf{x}^{\prime}=\mathbf{d}+\mathbf{A} \mathbf{x}, \quad \mathbf{x}=\mathbf{A}^{T}\left(\mathbf{x}^{\prime}-\mathbf{d}\right),
$$

where

$$
\mathbf{A}=\left(\begin{array}{rr}
\cos \theta & \sin \theta \\
-\sin \theta & \cos \theta
\end{array}\right), \quad \mathbf{A}^{T}=\mathbf{A}^{-1}=\left(\begin{array}{rr}
\cos \theta & -\sin \theta \\
\sin \theta & \cos \theta
\end{array}\right) .
$$

Differentiating (3) we obtain the velocity in the moving frame of reference

$$
\mathbf{v}=\dot{\theta} \mathbf{I}_{0} \mathbf{x}+\mathbf{A}^{T}\left(\mathbf{v}^{\prime}-\dot{\mathbf{d}}\right), \quad \mathbf{I}_{0}=\left(\begin{array}{rr}
0 & -1 \\
1 & 0
\end{array}\right) .
$$

In the above derivation we have applied the relations

$$
\dot{\mathbf{A}}^{T}=-\dot{\theta}\left(\begin{array}{rr}
\sin \theta & \cos \theta \\
-\cos \theta & \sin \theta
\end{array}\right), \quad \dot{\mathbf{A}}^{T} \mathbf{A}=\dot{\theta} \mathbf{I}_{0} .
$$

Throughout this paper we will use the superscript dot to denote differentiation with respect to time. The velocity in the absolute frame of reference is therefore related to the moving frame of reference by

$$
\mathbf{v}^{\prime}=\mathbf{A}\left(\mathbf{v}-\dot{\theta} \mathbf{I}_{0} \mathbf{x}\right)+\dot{\mathbf{d}} .
$$

Using the above relations, we can derive the expressions for temporal and spatial operators in the moving frame of reference. First, from (3), we know

$$
\begin{aligned}
& \frac{\partial}{\partial x^{\prime}}=\frac{\partial}{\partial x} \frac{\partial x}{\partial x^{\prime}}+\frac{\partial}{\partial y} \frac{\partial y}{\partial x^{\prime}}=\cos \theta \frac{\partial}{\partial x}+\sin \theta \frac{\partial}{\partial y} \\
& \frac{\partial}{\partial y^{\prime}}=\frac{\partial}{\partial x} \frac{\partial x}{\partial y^{\prime}}+\frac{\partial}{\partial y} \frac{\partial y}{\partial y^{\prime}}=-\sin \theta \frac{\partial}{\partial x}+\cos \theta \frac{\partial}{\partial y}
\end{aligned}
$$

and this implies

$$
\nabla^{\prime}=\mathbf{A} \nabla, \quad\left(\nabla^{\prime}\right)^{2}=(\nabla)^{2} .
$$

Therefore equation (5) plus (7) leads to

$$
\nabla^{\prime} \cdot \mathbf{v}^{\prime}=\nabla \cdot \mathbf{v}, \quad \nabla^{\prime} p=\mathbf{A} \nabla p, \quad\left(\nabla^{\prime}\right)^{2} \mathbf{v}^{\prime}=\mathbf{A} \nabla^{2} \mathbf{v} .
$$

Furthermore, the nonlinear term in the moving frame of reference can be derived, using equation (5) and (7) as

$$
\begin{aligned}
\left(\mathbf{v}^{\prime} \cdot \nabla^{\prime}\right) \mathbf{v}^{\prime}= & {\left[\left(\mathbf{A}\left(\mathbf{v}-\dot{\theta} \mathbf{I}_{0} \mathbf{x}\right)+\dot{\mathbf{d}}\right) \cdot \mathbf{A} \nabla\right]\left(\mathbf{A}\left(\mathbf{v}-\dot{\theta} \mathbf{I}_{0} \mathbf{x}\right)+\dot{\mathbf{d}}\right) } \\
= & \mathbf{A}\left[\left(\mathbf{v}-\dot{\theta} \mathbf{I}_{0} \mathbf{x}+\mathbf{A}^{T} \dot{\mathbf{d}}\right) \cdot \nabla\right]\left(\mathbf{v}-\dot{\theta} \mathbf{I}_{0} \mathbf{x}\right) \\
= & \mathbf{A}\left[(\mathbf{v} \cdot \nabla) \mathbf{v}-\left(\left(\dot{\theta} \mathbf{I}_{0} \mathbf{x}-\mathbf{A}^{T} \dot{\mathbf{d}}\right) \cdot \nabla\right) \mathbf{v}-\dot{\theta} \mathbf{I}_{0} \mathbf{v}\right. \\
& \left.-(\dot{\theta})^{2} \mathbf{x}-\dot{\theta} \mathbf{I}_{0} \mathbf{A}^{T} \dot{\mathbf{d}}\right],
\end{aligned}
$$

where we note that $\mathbf{v} \cdot \nabla\left(\dot{\theta} \mathbf{I}_{0} \mathbf{x}\right)=\dot{\theta} \mathbf{I}_{0} \mathbf{v}$ and $\mathbf{I}_{0} \mathbf{I}_{0}=-\mathbf{I}$. Finally, because

$$
\left(\frac{\partial}{\partial t}\right)_{a}=\frac{\partial}{\partial x} \frac{\partial x}{\partial t}+\frac{\partial}{\partial y} \frac{\partial y}{\partial t}+\left(\frac{\partial}{\partial t}\right)_{r}
$$


where the subscripts ' $a$ ' and ' $r$ ' refer to the absolute and relative (moving) frames of reference respectively, we have

$$
\left(\frac{\partial \mathbf{v}^{\prime}}{\partial t}\right)_{a}=\left(\mathbf{v}_{c} \cdot \nabla\right) \mathbf{v}^{\prime}+\left(\frac{\partial \mathbf{v}^{\prime}}{\partial t}\right)_{r}
$$

where

$$
\mathbf{v}_{c}=\left(\frac{\partial \mathbf{x}}{\partial t}\right)_{a}=\dot{\mathbf{A}}^{T}\left(\mathbf{x}^{\prime}-\mathbf{d}\right)-\mathbf{A}^{T} \dot{\mathbf{d}}=\dot{\theta} \mathbf{I}_{0} \mathbf{x}-\mathbf{A}^{T} \dot{\mathbf{d}} .
$$

Therefore substituting equations (5) and (11) into equation (10) we find

$$
\begin{aligned}
\left(\frac{\partial \mathbf{v}^{\prime}}{\partial t}\right)_{a}= & {\left[\left(\dot{\theta} \mathbf{I}_{0} \mathbf{x}-\mathbf{A}^{T} \dot{\mathbf{d}}\right) \cdot \nabla\right]\left[\mathbf{A}\left(\mathbf{v}-\dot{\theta} \mathbf{I}_{0} \mathbf{x}+\mathbf{A}^{T} \dot{\mathbf{d}}\right)\right] } \\
& +\frac{\partial}{\partial t}\left(\mathbf{A}\left(\mathbf{v}-\dot{\theta} \mathbf{I}_{0} \mathbf{x}\right)+\dot{\mathbf{d}}\right)_{r} \\
= & \mathbf{A}\left[\left(\dot{\theta} \mathbf{I}_{0} \mathbf{x}-\mathbf{A}^{T} \dot{\mathbf{d}}\right) \cdot \nabla\right] \mathbf{v}+(\dot{\theta})^{2} \mathbf{x}+\dot{\theta} \mathbf{I}_{0} \mathbf{A}^{T} \dot{\mathbf{d}} \\
& +\dot{\mathbf{A}}\left(\mathbf{v}-\dot{\theta} \mathbf{I}_{0} \mathbf{x}\right)+\ddot{\mathbf{d}}+\mathbf{A}\left(\left(\frac{\partial \mathbf{v}}{\partial t}\right)_{r}-\ddot{\theta} \mathbf{I}_{0} \mathbf{x}\right) \\
= & \mathbf{A}\left\{\left(\frac{\partial \mathbf{v}}{\partial t}\right)_{r}+\left[\left(\dot{\theta} \mathbf{I}_{0} \mathbf{x}-\mathbf{A}^{T} \dot{\mathbf{d}}\right) \cdot \nabla\right] \mathbf{v}+\dot{\theta} \mathbf{I}_{0} \mathbf{A}^{T} \dot{\mathbf{d}}\right. \\
& \left.-\dot{\theta} \mathbf{I}_{0} \mathbf{v}+\mathbf{A}^{T} \ddot{\mathbf{d}}-\ddot{\theta} \mathbf{I}_{0} \mathbf{x}\right\},
\end{aligned}
$$

where we have used the following relations

$$
\mathbf{A} \mathbf{A}^{T}=\mathbf{I}, \quad \dot{\mathbf{A}}=-\dot{\theta} \mathbf{A} \mathbf{I}_{0}, \quad \mathbf{I}_{0} \mathbf{I}_{0}=-\mathbf{I}, \quad(\mathbf{v} \cdot \nabla) \mathbf{x}=\mathbf{v} .
$$

Now substituting (8),(9) and (12) into the Navier Stokes equations (1) leads to the Navier Stokes equations in the moving frame of reference

$$
\begin{aligned}
\nabla \cdot \mathbf{v} & =0 \\
\frac{\partial \mathbf{v}}{\partial t}+(\mathbf{v} \cdot \nabla) \mathbf{v} & =-\nabla p+\nu \nabla^{2} \mathbf{v}+\mathbf{G}(\mathbf{v}, t), \\
\mathbf{G}(\mathbf{v}, t) & =2 \dot{\theta} \mathbf{I}_{0} \mathbf{v}+(\dot{\theta})^{2} \mathbf{x}+\ddot{\theta} \mathbf{I}_{0} \mathbf{x}-\mathbf{A}^{T} \ddot{\mathbf{d}} .
\end{aligned}
$$

In addition, using equations (5) and (6) the vorticity is transformed as

$$
\omega^{\prime}=\frac{\partial v^{\prime}}{\partial x^{\prime}}-\frac{\partial u^{\prime}}{\partial y^{\prime}}=\frac{\partial v}{\partial x}-\frac{\partial u}{\partial y}-2 \dot{\theta}=\omega-2 \dot{\theta},
$$

which shows that the transformation does not alter the vorticity if the body has no rotation.

In fact, equations (13) are the same equations as typically adopted in geophysical flow [10], where the term $2 \dot{\theta} \mathbf{I}_{0} \mathbf{v}$ is related to the deflecting or Coriolis force and $(\dot{\theta})^{2} \mathbf{x}$ is related to the centrifugal force. However the terms $-\mathbf{A}^{T} \ddot{\mathbf{d}}$ and $\ddot{\theta} \mathbf{I}_{0} \mathbf{x}$ are related to the forces due to unsteady translation and rotation, which are not typically present in geophysical flow. Similar to the treatment in geophysical flow, we may also rewrite (13) without explicitly containing the coordinate $\mathbf{x}$, by modifying the pressure to include the Coriolis and centrifugal forces. However, 
we prefer to keep the pressure unaltered, since this not only simplifies the implementation of the pressure boundary condition for the splitting scheme in the moving frame of reference, but is convenient when coupling a structural equation which is primarily driven by the pressure forces in the absolute frame of reference.

In non-accelerating motion this formulation is effectively stating that the problem of a moving body in a uniform flow is equivalent to that of a stationary body in a moving flow. For example, when a body is fixed in a flow and forms an angle with the free stream velocity in the far-field, then according to the above formulation, $\dot{\theta}=0, \theta \neq 0$. We therefore need to solve a problem with a far-field velocity $\mathbf{v}=\mathbf{A} \mathbf{v}^{\prime}=(\cos \theta,-\sin \theta)^{T}$ with the no-slip condition applied on the surface of the body.

\subsection{Transformation of Neumann and Dirichlet Boundary Conditions}

The far-field Dirichlet boundary condition for the transformed Navier Stokes equations can be specified using equation (4), i.e.,

$$
\mathbf{v}=\dot{\theta} \mathbf{I}_{0} \mathbf{x}+\mathbf{A}^{T}\left(\mathbf{v}^{\prime}-\dot{\mathbf{d}}\right), \text { in the far-field, }
$$

where $\mathbf{v}^{\prime}$ is velocity in the far-field in the absolute frame of reference.

For many numerical schemes, a far-field Neumann boundary condition at the outflow is typically applied in the absolute frame of reference, such that

$$
\nabla^{\prime} u^{\prime} \cdot \mathbf{n}^{\prime}=g_{N}^{u^{\prime}}, \quad \nabla^{\prime} v^{\prime} \cdot \mathbf{n}^{\prime}=g_{N}^{v^{\prime}},
$$

where $\mathbf{n}^{\prime}$ is the outward normal to the boundary and $g_{N}^{u^{\prime}}, g_{N}^{v^{\prime}}$ are known functions. We therefore need to transform this condition into the moving frame of reference. Expanding the absolute frame of reference Neumann conditions in terms of the moving frame of reference variables we obtain

$$
\begin{aligned}
\nabla^{\prime} u^{\prime} \cdot \mathbf{n}^{\prime} & =\mathbf{A n} \cdot \mathbf{A} \nabla\left((u+\dot{\theta} y) \cos \theta+(v-\dot{\theta} x) \sin \theta+g^{\prime}(t)\right) \\
& =\left[\cos \theta \nabla u+\sin \theta \nabla v+\dot{\theta}(-\sin \theta, \cos \theta)^{T}\right] \cdot \mathbf{n}, \\
\nabla^{\prime} v^{\prime} \cdot \mathbf{n}^{\prime} & =\mathbf{A n} \cdot \mathbf{A} \nabla\left(-(u+\dot{\theta} y) \sin \theta+(v-\dot{\theta} x) \cos \theta+h^{\prime}(t)\right) \\
& =\left[-\sin \theta \nabla u-\cos \theta \nabla v+\dot{\theta}(-\cos \theta,-\sin \theta)^{T}\right] \cdot \mathbf{n} .
\end{aligned}
$$

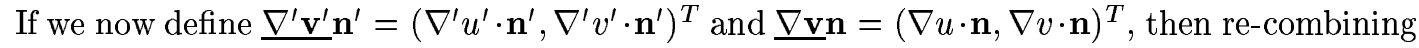
equation (16), we can show that

$$
\underline{\nabla^{\prime} \mathbf{v}^{\prime} \mathbf{n}^{\prime}}=[\mathbf{A}(\underline{\nabla \mathbf{v}})+\dot{\mathbf{A}}] \mathbf{n}=\mathbf{A}\left(\underline{\nabla \mathbf{v}}-\dot{\theta} \mathbf{I}_{0}\right) \mathbf{n}=\mathbf{A}\left[\left(\underline{\nabla \mathbf{v} \mathbf{n}}-\dot{\theta} \mathbf{I}_{0} \mathbf{n}\right] .\right.
$$

Furthermore, assume

$$
\mathbf{g}_{N}^{\prime}=\left(g_{N}^{u^{\prime}}, g_{N}^{v^{\prime}}\right)^{T}=\mathbf{A}\left(g_{N}^{u}, g_{N}^{v}\right)^{T}=\mathbf{A} \mathbf{g}_{N} .
$$

Then finally, combining equations (15),(17) and (18) we obtain the corresponding Neumann boundary conditions in the moving frame of reference

$$
\nabla u \cdot \mathbf{n}=g_{N}^{u^{\prime}}-\dot{\theta} n_{y}, \quad \nabla v \cdot \mathbf{n}=g_{N}^{v^{\prime}}+\dot{\theta} n_{x} .
$$




\subsection{Force and Moment Evaluation}

Before discussing the structural solver in section 2.4 we first need to evaluate the forces and moment acting on a body, which will be resolved into components parallel and perpendicular to the free stream velocity for use in the structural solver.

The aerodynamic force exerted on a body by the flow is the integration of local stress $\sigma=-p \mathbf{I}+\tau$, i.e.,

$$
\mathbf{F}=\int_{\Gamma(t)} \sigma \mathbf{n}^{\prime} d \mathbf{s}^{\prime}=-\int_{\Gamma(t)} p \mathbf{n}^{\prime} d \mathbf{s}^{\prime}+\int_{\Gamma(t)} \tau \mathbf{n}^{\prime} d \mathbf{s}^{\prime}=\mathbf{F}_{p}^{\prime}+\mathbf{F}_{\nu}^{\prime}
$$

where $\mathbf{n}^{\prime}$ is the outward unit normal on the body and $\mathbf{F}_{p}^{\prime}$ refers to the pressure force, $\mathbf{F}_{\nu}^{\prime}$ refers to the viscous force. The above integration is defined in the absolute frame of reference. However the total force can be evaluated in the transformed plane and then rotated back into the absolute frame of reference since

$$
\mathbf{F}=\mathbf{F}_{p}^{\prime}+\mathbf{F}_{\nu}^{\prime}=\mathbf{A}\left(\mathbf{F}_{p}+\mathbf{F}_{\nu}\right)
$$

where $\mathbf{F}_{p}$ and $\mathbf{F}_{\nu}$ are the forces calculated in the transformed plane.

The moment about a point $\mathbf{P}$ is defined in the absolute frame of reference as

$$
M^{\prime}=\int_{\Gamma(t)} \mathbf{r}^{\prime} \times\left(\sigma \cdot \mathbf{n}^{\prime}\right) d s^{\prime}=-\oint p\left(\mathbf{r}^{\prime} \times \mathbf{n}^{\prime}\right) d s^{\prime}+\oint \mathbf{r}^{\prime} \times\left(\tau \cdot \mathbf{n}^{\prime}\right) d s^{\prime}=M_{p}^{\prime}+M_{\nu}^{\prime}
$$

where $\mathbf{r}^{\prime}$ is a radius vector from point $\mathbf{P}$ to the element of the surface $\Gamma(t)$. In the current formulation we require the moment about the centre of the body and so $\mathbf{P}=\mathbf{d}$ and therefore if $\mathbf{x}^{\prime} \in \Gamma(t)$ then

$$
\mathbf{r}^{\prime}=\mathbf{x}^{\prime}-\mathbf{d}=\mathbf{d}+\mathbf{A} \mathbf{x}-\mathbf{d}=\mathbf{A} \mathbf{x}
$$

which simply states that $\mathbf{r}^{\prime}$ is the rotated vector from the origin in the moving frame of reference. Therefore for a two dimensional problem

$$
M^{\prime}=M=-\oint p(\mathbf{x} \times \mathbf{n}) d s+\oint \mathbf{x} \times(\tau \cdot \mathbf{n}) d s
$$

and so the calculation for the moment can also be evaluated in the moving frame of reference.

\subsection{Equation of Motion for the Body}

For a single rigid body, planar motion can be described in terms of three displacement components [9], $\mathbf{X}=(\zeta, \eta, \theta)^{T}$, where $\zeta$ and $\eta$ are the translational displacement components in $x^{\prime}$ and $y^{\prime}$ direction, respectively, and $\theta$ denotes the rotational displacement component. When relating to the transformed Navier Stokes equation and corresponding boundary conditions, we know that $\zeta=g(t), \eta=h(t)$ and $\theta=\theta(t)$. We may write the governing equation for this planar motion as

$$
\mathbf{M} \ddot{\mathbf{X}}+\mathbf{D} \dot{\mathbf{X}}+\mathbf{K X}=\overline{\mathbf{F}},
$$

where $\mathbf{M}, \mathbf{D}$ and $\mathbf{K}$ are the mass, damping and stiffness matrices respectively, and $\overline{\mathbf{F}}$ is a vector whose components are the external forces and moment acting on the body. The mounting 
system for the body is assumed to have both stiffness and damping. A simple mode of planar motion is where each one of the three degrees of freedom has a single spring and dashpot which are uncoupled from the other degrees of freedom. In this case, $\mathbf{M}, \mathbf{D}$ and $\mathbf{K}$ are all diagonal matrices with constant coefficients.

Equation (20) is a second order initial value problem for which there are many effective solution methods. In the current work, we have employed the trapezoidal scheme which is second order accurate in time.

$$
\begin{aligned}
\mathbf{X}^{n+1} & =\mathbf{X}^{n}+\frac{1}{2} \Delta t\left(\mathbf{V}^{n}+\mathbf{V}^{n+1}\right), \\
\mathbf{V}^{n+1} & =\mathbf{V}^{n}+\frac{1}{2} \Delta t\left(\mathbf{B}^{n}+\mathbf{B}^{n+1}\right) \\
\mathbf{M B}^{n+1} & +\mathbf{D V}^{n+1}+\mathbf{K} \mathbf{X}^{n+1}=\overline{\mathbf{F}}^{n+1}
\end{aligned}
$$

where $\mathbf{X}^{n}, \mathbf{V}^{n}$ and $\mathbf{B}^{n}$ are respectively numerical approximations for $\mathbf{X}, \dot{\mathbf{X}}$ and $\ddot{\mathbf{X}}$ at time $t_{n}=n \Delta t$. Notice that this scheme also requires the evaluation of the forcing term $\overline{\mathbf{F}}$ at the time level $n+1$. However for an explicit loosely coupled fluid structure interaction algorithm we require $\overline{\mathbf{F}}^{\mathbf{n}+\mathbf{1}}$ to be independent of $\mathbf{V}^{n+1}$ and so it cannot be directly evaluated. We therefore adopt an extrapolation/relaxation approximation such that

$$
\overline{\mathbf{F}}^{n+1} \approx \tilde{\mathbf{F}}^{n+1}=\vartheta \overline{\mathbf{F}}^{n}+(1-\vartheta) \overline{\mathbf{F}}^{n-1}
$$

The parameter $\vartheta$ can be used as either a relaxation or extrapolation parameter. If $\vartheta=\frac{3}{2}$ we have a second order extrapolation for $\overline{\mathbf{F}}^{n+1}$.

\subsection{Coupling Algorithm}

The loose coupling between the fluid and structure solver follows a computational procedure of the form:

Given the solution at time level $n$.

1. Calculate force and moment $\overline{\mathbf{F}}^{\mathbf{n}}$, and extrapolate the force and moment to the time level $t^{n+1}$ via the extrapolation/relaxation approximation $(22)$.

2. Solve the structural equation (21) for $\mathbf{X}^{n+1}, \mathbf{V}^{n+1}$ and $\mathbf{B}^{n+1}$.

3. Solve the Navier Stokes equations (13) from $t_{n}$ to $t_{n+1}$ using the velocity boundary conditions $\mathbf{V}^{n+1}$.

4a Let $n=n+1$ and go to step 1 .

Or alternatively

4b Sub-iterate to achieve an implicit value of $\tilde{\mathbf{F}}^{n+1}$. Denoting the current iteration level as $j$ we re-evaluate $\tilde{\mathbf{F}}_{j}^{n+1}$ based directly upon $\mathbf{V}_{j}^{n+1}$. When a convergence criterion of the form

$$
|| \tilde{\mathbf{F}}_{j+1}^{n+1}-\tilde{\mathbf{F}}_{j}^{n+1}|| /|| \tilde{\mathbf{F}}_{j+1}^{n+1} \mid<\epsilon
$$

is satisfied, then let $n=n+1$ and go to next time level, otherwise go to step 2. ( $\epsilon$ is a prescribed small constant.) 


\section{Navier-Stokes Spatial and Temporal Discretisation}

At this stage we have an algorithm for a loosely coupled fluid/structure interaction. The formulation so far is independent of the type of Navier Stokes solver and the spatial discretisation. In the following simulations we have adopted a high order splitting scheme [11] with a spectral/ $h p$ element spatial discretisation $[12,13]$.

\subsection{High Order Splitting Scheme}

The incompressible Navier-Stokes equations in the moving frame of reference can be written as

$$
\frac{\partial \mathbf{v}}{\partial t}=-\nabla p+\nu \boldsymbol{L}(\mathbf{v})+\boldsymbol{N}(\mathbf{v})
$$

where

$$
\begin{aligned}
\boldsymbol{L}(\mathbf{v}) & \equiv \nabla^{2} \mathbf{v} \\
\boldsymbol{N}(\mathbf{v}) & \equiv-\mathbf{v} \cdot \nabla \mathbf{v}+\boldsymbol{G}(\mathbf{v}, \mathbf{t})
\end{aligned}
$$

and $\mathbf{v}$ and $p$ denote the velocity vector and the pressure, respectively. The forcing term $\boldsymbol{G}(\mathbf{v}, t)$ is defined in equation (13c). Following [11] the numerical splitting scheme can be written in three steps as

$$
\begin{aligned}
\frac{\hat{\boldsymbol{v}}-\mathbf{v}^{\mathbf{n}}}{\Delta t} & =\sum_{q=0}^{J_{e}-1} \beta_{q} \boldsymbol{N}\left(\mathbf{v}^{\mathbf{n}-\mathbf{q}}\right) \\
\frac{\boldsymbol{v}^{\star}-\hat{\boldsymbol{v}}}{\Delta t} & =-\nabla \bar{p}^{n+1} \\
\frac{\boldsymbol{v}^{n+1}-\boldsymbol{v}^{\star}}{\Delta t} & =\nu \sum_{q=0}^{J_{i}-1} \gamma_{q} \boldsymbol{L}\left(\mathbf{v}^{\mathbf{n}+\mathbf{1}-\mathbf{q}}\right)
\end{aligned}
$$

In the first step, (23a), the non-linear advection and forcing terms are advanced using a convective form which is integrated in time using a multilevel Adams-Bashforth scheme denoted by the coefficients $\beta_{q}$. In the second step, the time-averaged pressure $\bar{p}$ is obtained by taking the divergence of (23b) and assuming $\nabla \cdot \mathbf{v}^{\star}=0$ to arrive at a Poisson equation which is supplemented with boundary conditions of the form

$$
\begin{aligned}
\frac{\partial p^{n+1}}{\partial \mathbf{n}} & =\mathbf{n} \cdot\left\{-\frac{\mathbf{v}^{n+1}-\mathbf{v}^{n}}{\Delta t}\right\} \\
& -\mathbf{n} \cdot\left\{\sum_{q=0}^{J_{e}-1} \beta_{q}[(\mathbf{v} \cdot \nabla) \mathbf{v}-\mathbf{G}+\nu \nabla \times(\nabla \times \mathbf{v})]^{n-q}\right\} .
\end{aligned}
$$

These boundary conditions ensure that the splitting error associated with the scheme is consistent with the overall temporal discretisation. Finally the viscous component is treated implicitly using either an Euler backwards difference or Crank-Nicolson scheme and step (23c) is re-arranged into a Helmholtz equation for each velocity component. At this stage the unsteady far field boundary conditions given by equations (14) and (19) are imposed. 


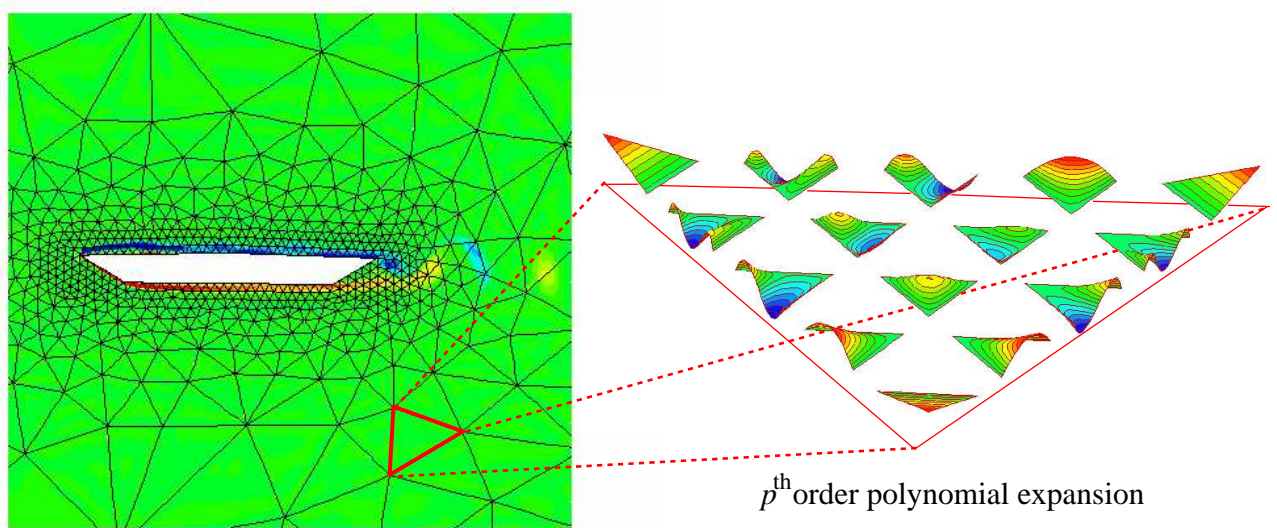

Discretisation into sub-domains of size $h$

Figure 2. Computational $h$-type domain highlighting the $p$-type two dimensional expansion basis

\subsection{Spectral/hp Element Discretisation}

The spatial discretisation adopted in the current work uses a spectral/ $h p$ element technique as detailed in $[12,13]$. As shown in figure 2, in this approach the solution domain is decomposed into triangular subdomains similar to the finite element and finite volume methods. Then within each subdomain we expand the solution using an arbitrary polynomial expansion or order $p$. The figure shows a computational domain subdivided into a series of unstructured triangular domains. Within each sub-domain a polynomial expansion of arbitrary order is applied. In this figure we see all the polynomial modes necessary for a $P=4$ order expansion.

\section{Validation and Flow Simulation}

It is often convenient to consider the non-dimensional form of the Navier Stokes equations

$$
\begin{aligned}
\nabla^{*} \cdot \mathbf{v}^{*} & =0 \\
\frac{\partial \mathbf{v}^{*}}{\partial t^{*}}+\left(\mathbf{v}^{*} \cdot \nabla^{*}\right) \mathbf{v}^{*} & =-\nabla^{*} p^{*}+R e^{-1}\left(\nabla^{*}\right)^{2} \mathbf{v}^{*}
\end{aligned}
$$

The variables above are non-dimensionalised as,

$$
\begin{aligned}
t^{*}=\frac{t U}{D}, \quad x^{*} & =\frac{x}{D}, \quad y^{*}=\frac{y}{D}, \\
u^{*}=\frac{u}{U}, \quad v^{*} & =\frac{v}{U}, \quad p^{*}=\frac{p}{\rho U^{2}}, \\
R e=\frac{\rho U D}{\mu} & =\frac{U D}{\nu},
\end{aligned}
$$


where $D$ is a characteristic length, and $\rho$ and $U$ are the free stream's density and velocity. Correspondingly, the forces and moment are converted into

$$
F_{x}^{*}=\frac{F_{x}}{\rho D U^{2}}, \quad F_{y}^{*}=\frac{F_{y}}{\rho D U^{2}}, \quad M_{\theta}^{*}=\frac{M_{\theta}}{\rho D^{2} U^{2}} .
$$

where we note that $C_{L}=2 F_{x}^{*}, C_{D}=2 F_{y}^{*}$ and $C_{M}=2 M_{\theta}^{*} / 2$. For a specific problem, the corresponding structural equations should also be non-dimensionalised in terms of the above definitions. In general, if we denote the vibration amplitude for a particular body by $A$, then the dimensionless amplitude of flow-induced vibration $A / D$ can be regarded as a function of the four dimensionless parameters:

1. Reynolds number $R e$ defined in (25);

2. Reduced velocity $U_{r e d}=\frac{U}{f_{n} D}$, where $f_{n}$ is the frequency of vibration;

3. Mass ratio $n=\frac{m}{\rho D^{2}}$, where $m$ is the mass per unit length of the body, and

4. damping factor $\xi$.

When considering bodies of rectangular cross section we also require the thickness ratio $\Lambda=L / D$, where $L$ and $D$ are the length and depth of the model respectively. Note that in the current work we have considered the depth of the body to be the characteristic length.

In the following sections we will demonstrate a range of fluid structure interaction problems involving free and forced motions. In all simulations we have considered rectangular domains attached to the body motion. The Therefore the left top and bottom boundaries are treated as inflow type boundaries with Dirichlet velocity conditions. The right boundary is treated as an outflow boundary where $g_{N}^{u^{\prime}}=g_{N}^{v^{\prime}}=0$ and pressure in the absolute frame of reference is taken to be constant. We note that strictly speaking when considering a rotating frame of reference the inflow and outflow characteristic of the upper and lower boundaries will change during an oscillatory cycle. We have therefore considered upper and lower boundaries that are well removed from the region of interest in term of convective length scales.

\subsection{Translating motion}

In this section we will consider forced vertical oscillations and free oscillations for both vertical and in-line motion. These types of oscillations have previously been investigated by a variety of researchers and so provide a good benchmark to validate the implementation for translational motion.

In all of the following simulations a mesh of 1000 triangular elements as shown in figure 3 was used with a polynomial order of $p=8$.

4.1.1. Forced vertical oscillation When a bluff body is forced to oscillate in a sinusoidal manner under the prescribed motion of

$$
y(t)=A \cos (2 \pi f t),
$$

the frequency of vortex shedding can be controlled over a limited range of reduced velocities. This phenomenon is usually known as lock-in, where the vortex shedding frequency and the body oscillation frequency are synchronized. Both experiments and numerical simulation show that lock-in occurs only above a threshold amplitude of oscillation and that the lock-in range increases with increasing amplitude. Interestingly, in the lock-in range increasing the oscillation 


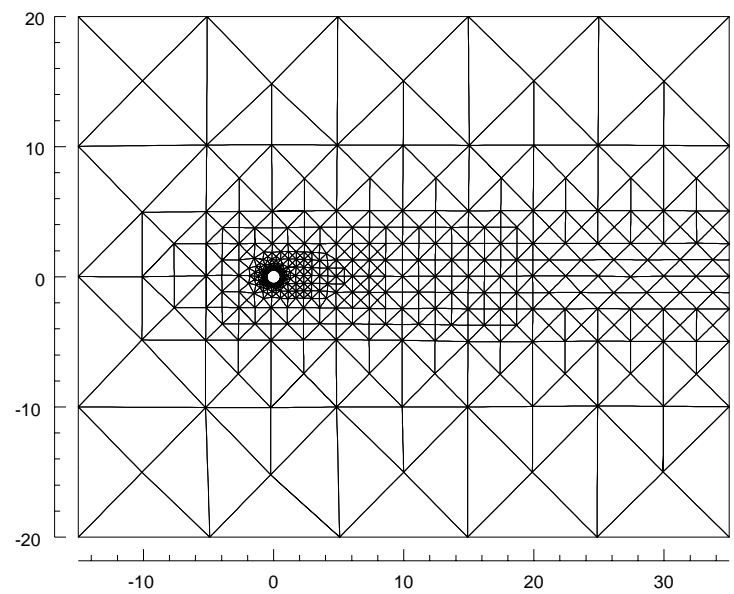

(a)

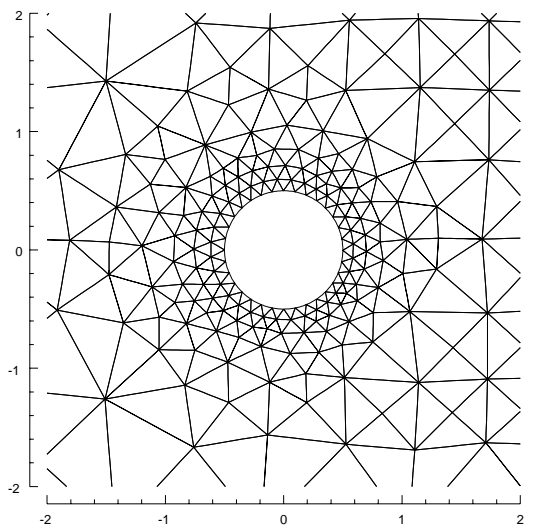

(b)

Figure 3. Computational mesh for flow past a translating cylinder using 1000 triangular regions. (a) whole domain (b) local resolution around cylinder.

amplitude can lead to dramatic changes in the flow patterns. Here, we present several cases for two-dimensional simulations at a Reynolds numbers of 200 to illustrate the phenomenon and demonstrate the capability of the algorithm discussed in section 2 .

An initial run using the spectral element method was performed for a fixed circular cylinder at Reynolds number 200 and a Strouhal number $\left(f_{s} D / U\right)$ of 0.198 was obtained, where $f_{s}$ is the vortex shedding frequency. As illustrated in figure 4, we then considered simulations of a circular cylinder undergoing forced oscillations at amplitudes of $A / D=0.5,0.6,0.65$ and frequency $f / f_{s}=0.8$. The flow is considered locked when the frequency of the lift force, $f_{L}$, is the same as the frequency of oscillation $f$ and for all three cases, i.e. $f_{L}=f=0.158$ (see $[14,15]$ for comparison).

For the smallest oscillation amplitude $(A / D=0.5)$, lock-in occurs and a von-Karman street type pattern if formed with two vortices, of opposite circulation, being shed in each cycle. As the amplitude of oscillation increases to 0.6, the mode of shedding changes and one pair of vortices with opposite circulation plus a single vortex are shed per cycle. This phenomenon has previously been observed in flow visualisations carried out by Griffin \& Ramberg [16], and by Williamson \& Roshko[17]. The latter authors denoted this flow pattern as the P+S mode. 


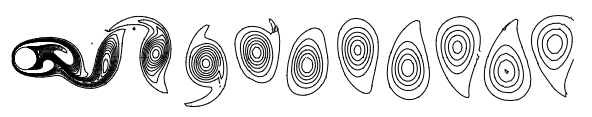

(a)

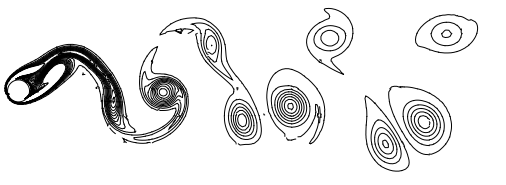

(c)

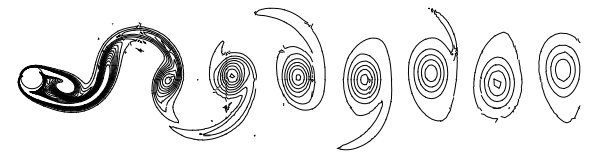

(b)

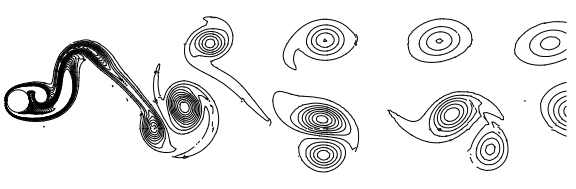

(d)

Figure 4. Vorticity contours for a circular cylinder at a Reynolds number of 200. (a) fixed cylinder with a Strouhal number of 0.1978. (b) $A / D=0.50$ and $f / f_{s}=0.8$. (c) $A / D=0.60$ and $f / f_{s}=0.8$. (d) $A / D=0.65$ and $f / f_{s}=0.8$.

\subsection{Free vertical oscillation}

The governing equation for one degree of freedom vertical motion or heaving mode is

$$
m \ddot{y}+c \dot{y}+k y=F_{y}(t),
$$

where $m$ is the mass per unit length of the body, $c$ is the damping coefficient, $k$ is the stiffness coefficient, and $y$ denotes the transverse displacement of the body centroid. The right-hand side of equation (26) is the vertical component of the aerodynamic force in the absolute coordinate system. If we use the same non-dimensionalised scales as applied to the Navier-Stokes equations, then we obtain

$$
\ddot{y}^{*}+2 \xi\left(\frac{2 \pi}{U_{r e d}}\right) \dot{y}^{*}+\left(\frac{2 \pi}{U_{r e d}}\right)^{2} y^{*}=\frac{F_{y}^{*}}{n},
$$

where

$$
U_{\text {red }}=\frac{U}{f_{n} D}(\text { reduced velocity }), \quad n=\frac{m}{\rho D^{2}} \quad(\text { mass ratio }) .
$$

Here $f_{n}$ is the natural frequency of the heaving mode of the structure.

Anagnostopoulos and Bearman [18] conducted a series of experiments for vortex-induced, cross-flow oscillations of a circular cylinder, in which the Reynolds number ranged between 90 and 150, a regime where the vortex street of a fixed cylinder is fully laminar. This experiment has become a test case for a range of numerical codes treating fluid/structure interaction problems $[1,2,3,19]$ although attempts to duplicate the lock-in range of the experiment have led to a noticeable discrepancy between numerical investigators. Bearing this in mind we have considered a few cases in the lock-in range. 

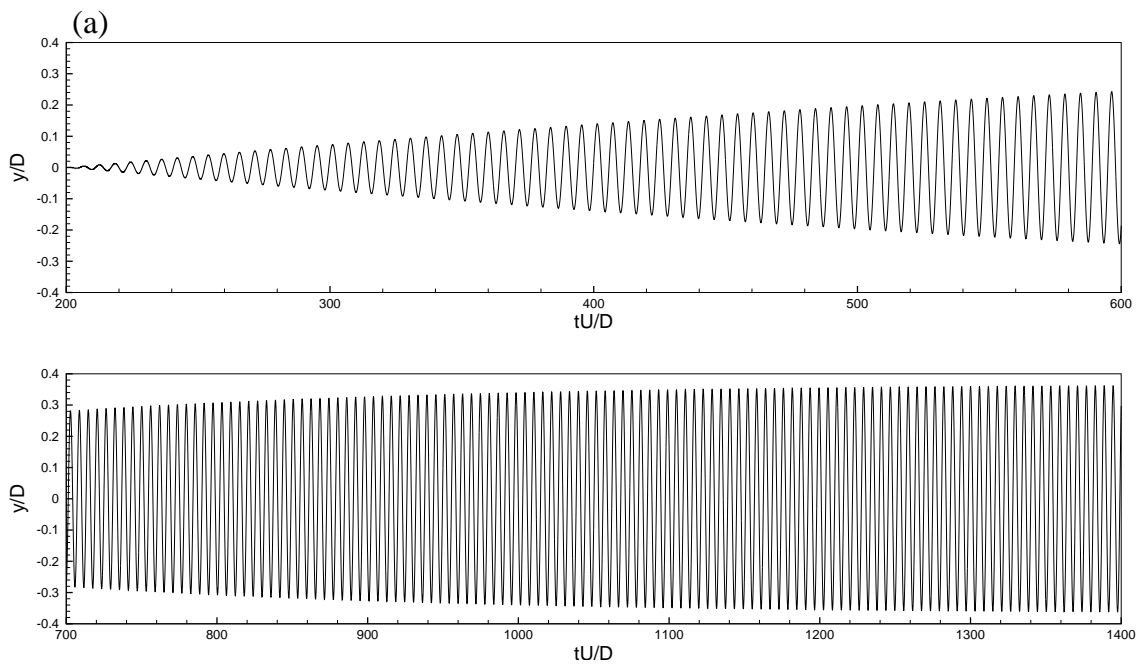

(b)
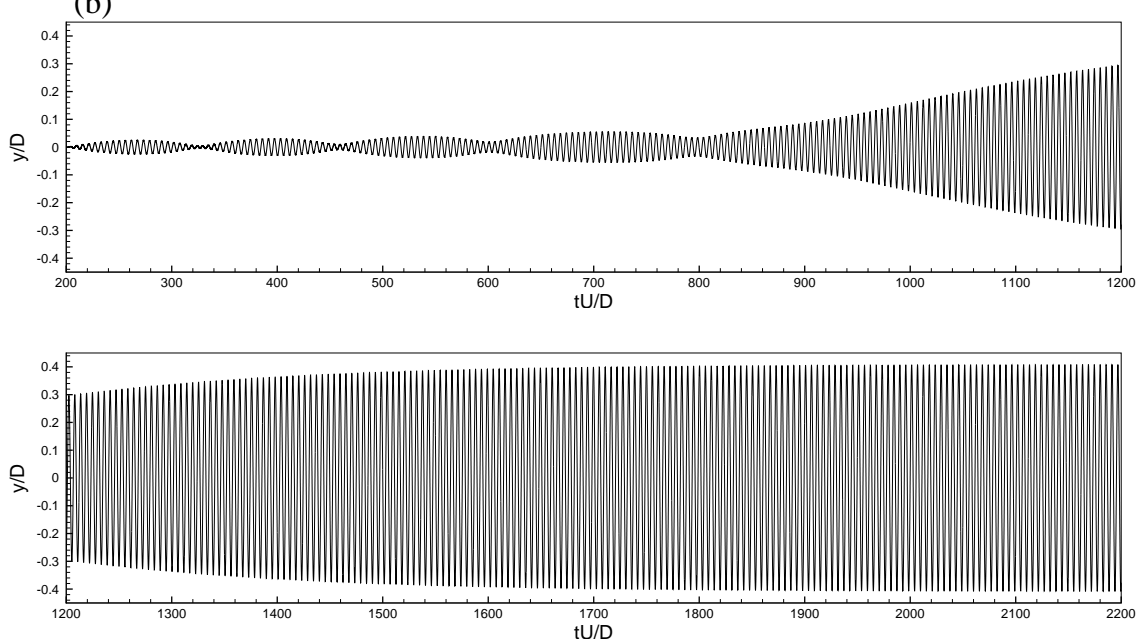

(c)

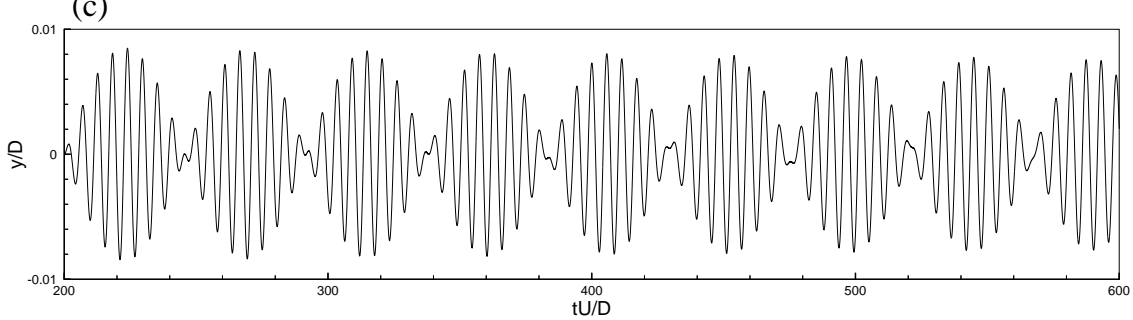

Figure 5. Displacement history of vertically freely vibrating cylinder with structural parameters $n=117.10, \xi=0.0012$. (a) $R e=106, U_{r e d}=5.88, f_{c}=0.169, f_{v}=0.169, f_{s}=0.17$. (b) $R e=100$, $U_{\text {red }}=5.577, f_{c}=0.179, f_{v}=0.179, f_{s}=0.1793$. (c) $R e=95$. 
The cylinder was constrained to move in the transverse direction and initally held rigid until the flow was fully developed at the desired Reynolds number. Validation runs where the cylinder was initally free to vibrate were also performed with no significant difference in the results.

The experimental values of the mass ratio and damping coefficients were 117.10 and 0.0012 , respectively. For a Reynolds number of 106 and $U_{\text {red }}=5.88$, we observed a monotonically growing oscillation amplitude until it reaches a limiting amplitude of about $37 \%$ of the cylinder diameter as shown in figure 5(a). For this case, the cylinder vibration and shedding frequency was $f_{c}=f_{L}=1 / 5.92$ as compared to the Strouhal frequency for a fixed cylinder of $f_{s}=1 / 5.88$. Reducing the Reynolds number to 100 with a corresponding reduced velocity of $U_{\text {red }}=5.58$ leads to a larger maximum oscillation amplitude of 0.42 as shown in figure $5(\mathrm{~b})$. When the Reynolds number is further reduced to 95 , the lock-in ceases to occur and the amplitude of oscillation is very small and modulated as shown in figure $5(\mathrm{c})$.

Within the lock-in range the trend that vibration amplitude increases as the Reynolds number is reduced is consistent with the experiments. However the magnitude of the vibration when compared with the experiments was smaller and the lock-in range in terms of Reynolds number occurred at lower values. We note that these discrepancies are consistent with other numerical predictions $[1,2,3]$.

\subsection{Free in-line oscillation}
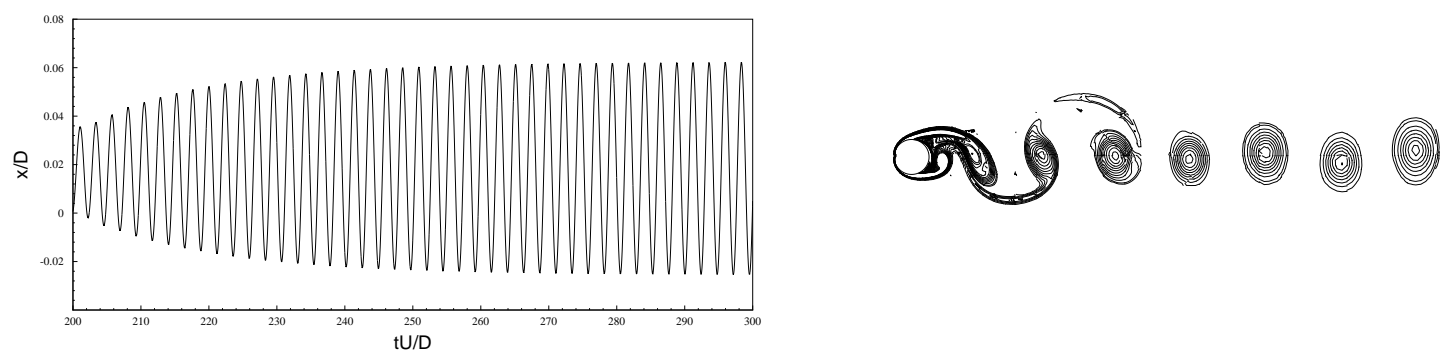

Figure 6. In-line response and instantaneous vorticity contours for a circular cylinder: $R e=400, n=$ $10, \xi=0$.

For a one degree of freedom in-line mode, the structural equation (20) becomes

$$
m \ddot{x}+c \dot{x}+k x=F_{x}(t),
$$

where $m$ is the mass per unit length of the body, $c$ and $k$ are the same as previously defined and $x$ denotes the in-line location of the body centroid. The right-hand side of equation (27) is taken to be the in-line component of the aerodynamic force. The non-dimensional form of (27) is

$$
\ddot{x}^{*}+2 \xi\left(\frac{2 \pi}{U_{r e d}}\right) \dot{x}^{*}+\left(\frac{2 \pi}{U_{r e d}}\right)^{2} x^{*}=\frac{F_{x}^{*}}{n} .
$$


For the case of a stationary circular cylinder at a Reynolds number of 400, we found the computed two-dimensional Strouhal number to be 0.218 . Choosing $U_{\text {red }}=2.2$ gives a natural structural frequency of 0.454 which is close to twice the Strouhal frequency. Setting the structural parameters $n=5, \xi=0$, the displacement of the freely vibrating cylinder and instantaneous vorticity contours are shown in figure 6 . The non-dimensional frequency of the oscillation is 0.436 , which is exactly twice the vortex shedding non-dimensional frequency of 0.218 .

\subsection{Rotational Motion}

In this section we will consider free and forced rotational oscillations around rectangular bodies with non-unit thickness ratios and therefore more relevant to fluid structure interaction around bridge deck structures. Bodies with high aspect ratios are far more susceptible to rotational galloping instabilities and it is the ability to capture this type of instability which has motivated the current work. In a complementary paper [20] we have investigated the rotational galloping in greater depth. In this section we will demonstrate the capability of the algorithm to capture rotational vibrations.

4.4.1. Forced rotation As a numerical experiment, the prescribed motion of rotation,

$$
\theta(t)=\Phi \cos (2 \pi f t)
$$

was imposed on a rectangular cross-section cylinder with thickness ratio of $L / D=5$ at different frequencies $f=0.025,0.05$ and 0.1 . The Reynolds number 250 based on the depth $D$ and a value of $\Phi=0.1$ was set which corresponding to a rotation of $\theta(t)$ in the range $\pm 5.7^{\circ}$. The computations were performed at a polynomial order of $p=8$ on a discretisation using 1261 triangular elements over the region $-25 D<x<55 D,-25 D<y<25 D$ where the body was centred at the origin.

Vorticity distributions for the different forced rotational frequencies are plotted in figure 7. In figure $7(\mathrm{~d})$ we have also plotted the case of $f=0.1$ with the computational mesh. In this figure we see that although the computational mesh has been rotated the wake vortices are being transported in the horizontal free-stream direction.

For a fixed rectangular body at zero angle of attack the Strouhal frequency based on the height of the body was $f_{s}=0.156$. Therefore the frequency ratio for cases (a),(b) and (c) are $f / f_{s}=0.16,0.32$ and 0.64 respectively. From the lift history it is possible to determine that in case (a) the shedding frequency is forced to lock into a $1 / 6^{\text {th }}$ of the body oscillation period. In case (b) the body oscillation forces the shedding frequency to lock into $1 / 3^{r d}$ of a body period and in case (c) the shedding period is locked directly into the body oscillation period. In cases (a) and (b) a vortex pairing processes is also evident which might be expected since the formation of the vortices is occurring at an offset position as the body rotates at a slower period. It is however unlikely that this doubling could be sustained under full three-dimensional flow conditions.

4.4.2. Free rotation For the one degree of freedom of rotational mode, equation (20) becomes

$$
I_{\theta} \ddot{\theta}+c_{\theta} \dot{\theta}+k_{\theta} \theta=M_{\theta}
$$




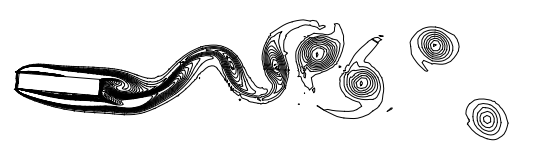

(a)

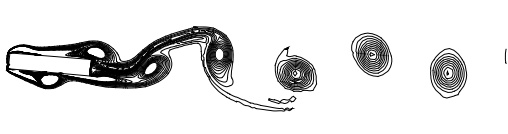

(c)

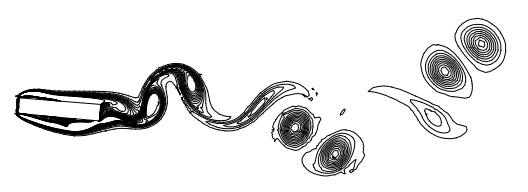

(b)

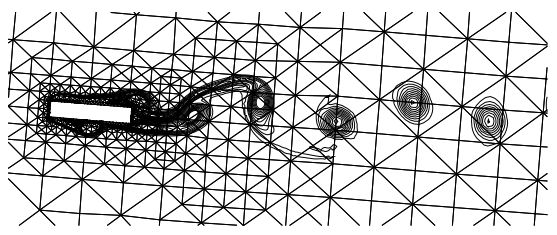

(d)

Figure 7. Forced rotation: $R e=250, \Phi=0.1$. (a) $f=0.025$. (b) $f=0.05$. (c) $f=0.1$.

where $I_{\theta}$ is the mass moment of inertia, $c_{\theta}$ is the damping coefficient, $k_{\theta}$ is the stiffness coefficient, and $\theta$ denotes the rotational angle of the body around the elastic centre. The righthand side of equation (20) is the moment relative to elastic centre. The non-dimensional form of equation (20) is

$$
\ddot{\theta}+2 \xi_{\theta}\left(\frac{2 \pi}{U_{\text {red }}}\right) \dot{\theta}+\left(\frac{2 \pi}{U_{\text {red }}}\right)^{2} \theta=\frac{M^{*}}{n}
$$

where

$$
U_{\text {red }}=\frac{U}{f_{n} D}(\text { reduced velocity }), \quad n=\frac{I_{\theta}}{\rho D^{4}}(\text { moment of inertia ratio }) .
$$

As an example of a freely rotating body we again consider the rectangular cross section body of thickness ratio $\Lambda=5$ at a Reynolds number of $R e=250$ based on depth. We have arbitrarily chosen the structural parameters to be $n=400, \xi_{\theta}=0.6$ and $U_{\text {red }}=40$. At this high reduced velocity the natural structural frequency is $1 / 40=0.025$ which is an order of magnitude smaller than the vortex shedding frequency.

In figure 8 we show the computational domain using 1261 elements rotated into the absolute frame of reference at a non-dimensional time of $t U / D=906$. From figure 9 (a) we see that this time corresponds to a rotation angle of $\theta=0.166 \mathrm{rads} .=9.5^{\circ}$ which is close to its maximum rotation.

Also shown in figure 8 are the instantaneous vorticity contours produced using a polynomial order of $p=6$. From figure 8 we see that the wake vortices are being transported primarily in the direction of the free stream which is aligned with the $\mathrm{x}$-axis. However at this level of rotation the wake vortices are approaching the upper right outer boundary and there is evidence of an interaction with the computational boundary. Clearly the use of a expanding upper and lower boundary would help alleviate this problem. However the location and concentration of this vorticity is very far from the body.

After a non-dimensional time of approximately 200 units we see from figure 9 that a rotational fluid structure interaction is established. The limit cycle of the rotation has a 

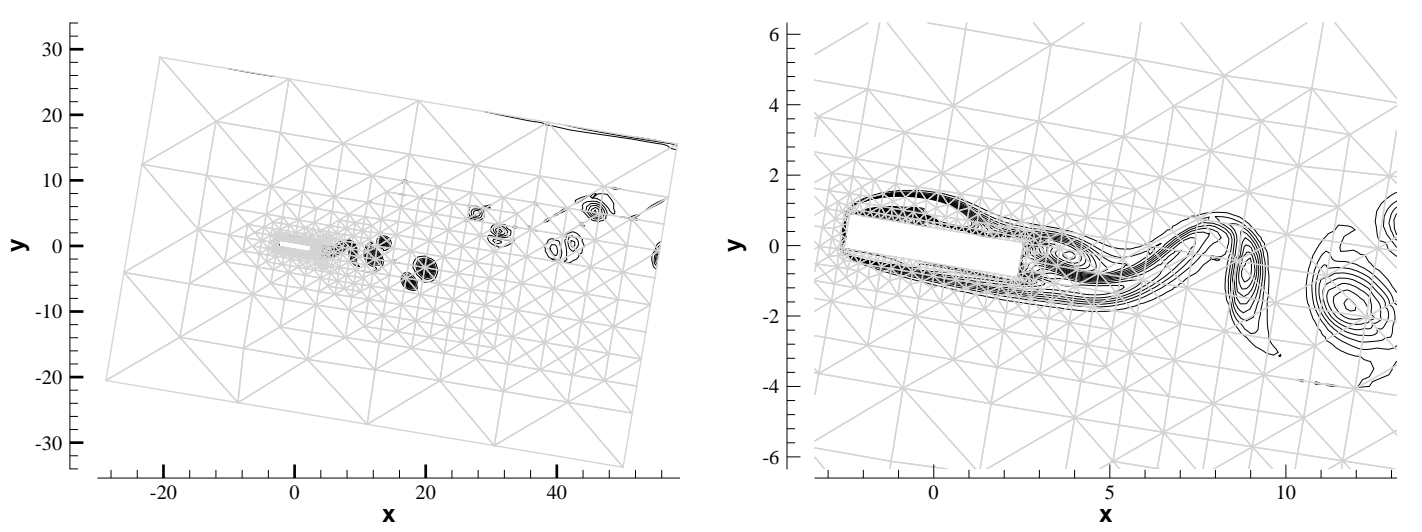

Figure 8. Free rotation of $\Lambda=5$ thickness ratio rectangular body at $t=906$. Structural parameters were $n=400, U_{\text {red }}=40, \xi_{\theta}=0.6$.

galloping instability frequency of $\approx 1 / 70$ which is much lower than the shedding frequency of $\approx 1 / 6$ and is to be expected from the high reduced velocity of the computation. Also shown in figure $9(\mathrm{~b})$ are the limit cycle lift, drag and moment coefficients as a function of time. These coefficients have been normalised based upon the depth of the body.

\section{Conclusion}

We have presented a complete derivation of the transformed Navier Stokes equations into a rotating and translating moving frame of reference attached to a rigid body. This transformation allows us to solve the coupled fluid/structural equations in a loosely coupled algorithm. The flexibility of this formulation combined with the high accuracy of spectral element discretisation provides an efficient tool for fundamental research and application on the fluid/structure interaction problems. Although the formulation has been derived for two dimensional problems, in principle, it can be generalised to three dimensional ones. One straightforward extension is to permit $\theta=\theta(z, t)$, and to use a Fourier expansion in the third direction. The resulting model can then include twisting and bending of the structure.

In the flow simulation, the lock-in phenomenon was captured for both heaving,in-line and rotational motion. The simulation results have shown a good agreement with both experimental and other existing numerical results. 


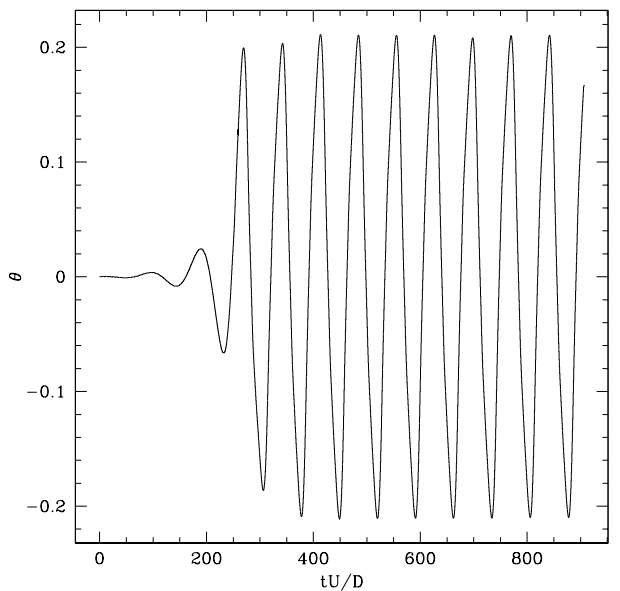

(a)
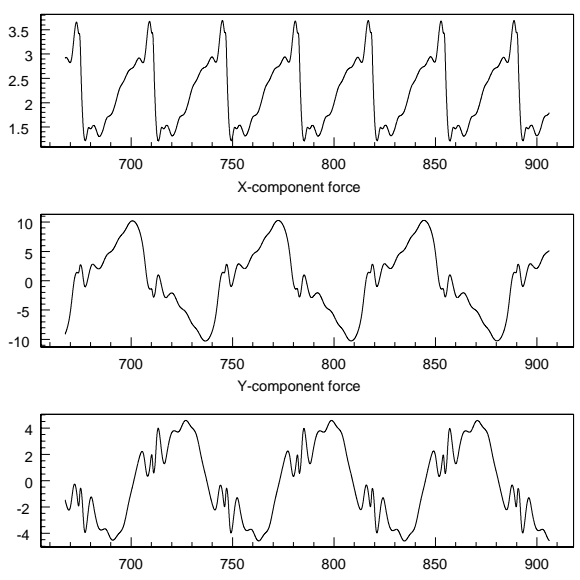

(b)

Figure 9. (a) Time evolution of angle of attach of body in radians. (b) Forces coefficient history normalised by depth.

\section{Acknowledgments}

The work is support by EPSRC under grant GL/L48096. The authors would like to acknowledge the support and advice of Simon Coleman of BMT Fluid Mechanics Ltd and Brian Smith and David MacKenzie of Flint and Neill Partnership.

\section{REFERENCES}

1. T. Nomura. Finite element analysis of vortex-induced vibrations of bluff cylinders. Journal of Wind Engineering and Industrial Aerodynamics, 46:587-594, 1993.

2. R. Wei, A. Sekine, and M. Shimura. Numerical analysis of $2 \mathrm{~d}$ vortex-induced oscillations of a circular cylinder. Int. J. Num. Meth. Fluids, 21:993-1005, 1995.

3. K. Schulz and Y. Kallinderis. Unsteady flow structure interaction for incompressible flows using deformable hybrid grids. Journal of Computational Physics, 143:569-597, 1998.

4. D. Newman and G. E. Karniadakis. A direct numerical simulation study of flow past a freely vibrating cable. Journal of Fluid Mechanics, 344:95-136, 1988.

5. D. B. Steinman and S. R. Watson. Bridges and their builders. Dover, New York., 1957.

6. R. H. Scanlan. On the state of stability considerations for suspended-span bridges under wind. In Proceedings IUTAM-IAHR Symposium, pages 595-618, Karlsruhe, Germany, 1979.

7. R. H. Scanlan and J. J. Tomko. Airfoil and bridge deck flutter derivatives. Journal of the Engineering Mechanics Division, ASCE, 97:1717-173, 1971.

8. T.J.R. Hughes, W.K. Liu, and T.K. Zimmerman. Lagrangian-eulerian finite element formulation for incompressible viscous flows. Computer Methods in Applied Mechanics and Engineer, 29:329-349, 1981.

9. T. Nomura and T. J.R. Hughes. An arbitrary lagrangian-eulerian finite element method for interaction of fluid and a rigid body. Computer Methods in Applied Mechanics and Engineering, 95:115-138, 1992.

10. D. W. Moore. Homogeneous fluids in rotation: viscous effects, chapter Rotating fluids in geophysics. Academic press, 1978.

11. G. Karniadakis, M. Israeli, and S Orszag. High-order splitting methods for the incompressible navierstokes equations. Journal of Computational Physics, 97:414-443, 1991. 
12. G.E. Karniadakis and S.J. Sherwin. Spectral/hp Element Methods for CFD. Oxford University Press, 1999.

13. S.J. Sherwin and G.E. Karniadakis. A triangular spectral element method; applications to the incompressible Navier-Stokes equations. Comp. Meth. Appl. Mech. Eng., 123:189, 1995.

14. J. R. Meneghini and P. W. Bearman. Numerical simulation of high amplitude oscillatory flow about a circular cylinder. Journal of Fluids and Structures, 9:435-455, 1995.

15. H. M. Blackburn and R. D. Henderson. A study of two-dimensional flow past an oscillating cylinder. Journal of Fluids Mechanics, 385:255-286, 1999.

16. O. M. Griffin and S. E. Ramberg. The vortex-street wakes of vibrating cylinders. Journal of Fluid Mechanics, 66:553-576, 1974.

17. C. H. Williamson and A. Roshko. Vortex formation in the wake of an oscillating cylinder. Journal of Fluids and Structures, 2:355-381, 1988.

18. P. Anagnostopoulos and P. W. Bearman. Response characteristics of vortex-excited cylinder at low reynolds numbers. Journal of Fluids and Structures, 6:39-50, 1992.

19. P. Anagnostopoulos. Numerical investigation of response and wake characteristics of a vortex-excited cylinder in a uniform stream. Journal of Fluids and Structures, 8:367-390, 1994.

20. L. Li I. Robertson, S.J. Sherwin, and P.W. Bearman. Numerical study of the rotaional and translational galloping. Journal of Fluid and Structures, 2001. to be submitted. 